\title{
Passive Light-Weight Arm Exoskeleton: Possible Applications
}

\author{
Markus Puchinger, Nithin Babu Rajendra Kurup, \\ and Margit Gfoehler ${ }^{(\bowtie)}$ \\ TU Wien, Vienna, Austria \\ margit.gfoehler@tuwien.ac.at
}

\begin{abstract}
Upper extremity exoskeletons are useful for humans in different ways: for motor rehabilitation, as assistive devices, or for the reduction of workrelated loads on the musculoskeletal system. This paper describes the design of a passive modular and light-weight arm exoskeleton with gravity support and discusses possible fields of application. Tests, carried out with enabled gravity support show reduced muscle activations and forces compared to the same movements with disabled gravity support, indicting the effectiveness of the design.
\end{abstract}

\section{Introduction}

Exoskeletons were first described in animals as external, hard supporting structures for protection, support and defense [1]. In the last decades exoskeletons became more important for humans, used in rehabilitation or as assistive devices for physically injured persons, or to support the musculoskeletal system while carrying heavy loads or working in physically demanding postures as e.g. overhead work. Work-related musculoskeletal disorders in the upper extremity are an important issue in the modern workplace, and there is still a lack of assistive devices that do not restrict a worker's mobility, such as could be used in situations requiring a worker to move with an object (e.g. a car on an assembly line) or to be inside an object (e.g. an aircraft fuselage) [2]. Exoskeletons can be classified as active and passive orthoses. In active exoskeletons additional joint moments are generated by active actuators as electrical motors, passive exoskeletons mainly use gravity compensation mechanisms to enable movements with reduced muscular effort.

Here we present the RETRAINER passive lightweight upper limb exoskeleton with integrated adjustable gravity-compensation and a modular structure that facilitates easy adaption to different requirements. The effectiveness of the built-in gravity compensation is determined and possible applications beyond rehabilitation are discussed.

Research supported by European Union's Horizon 2020 research and innovation program under grant agreement No 64721 . 


\section{Materials and Methods}

\subsection{Exoskeleton}

The described upper limb RETRAINER exoskeleton is a modular, light-weight passive device with 5 degrees of freedom (DoF) [3]. Humeral rotation and wrist pro/supination are either controlled by residual muscle forces or locked at customized positions. Shoulder elevation in sagittal and frontal planes as well as elbow flexion are actuated by residual muscle forces and additionally by neuromuscular electrostimulation (NMES) if needed and can be locked at any chosen position during the movement. The exoskeleton consists of six basic modules - wrist module, elbow module, humeralrotation module, shoulder module, inclination module and mounting module (Fig. 1).

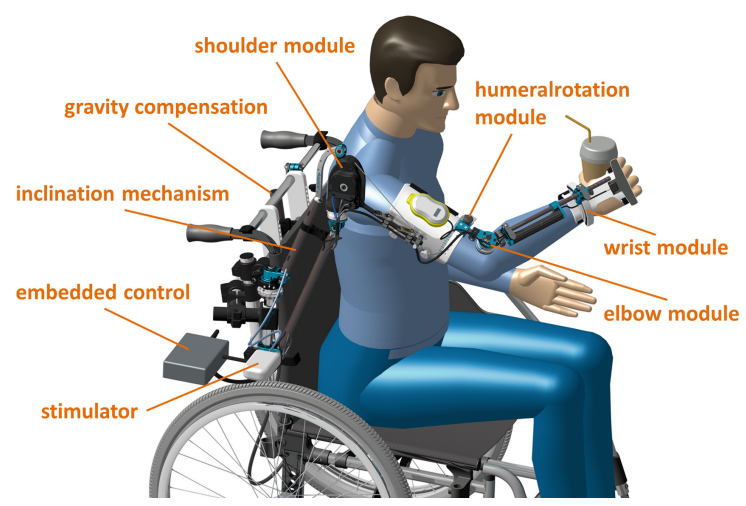

Fig. 1. Exoskeleton structure and modules: For stroke rehabilitation, the exoskeleton is mounted on a wheelchair and arm movements are facilitated through gravity compensation and if needed, additional actuation by NMES. Due to the flexible modular design single modules can be omitted if not needed.

The spring-based gravity compensation mechanisms integrated into the shoulder and elbow joint modules, provide compensation torques as a function of the respective joint angles.

Main element of the shoulder joint gravity compensation (Fig. 2) is a special alignment of rope pulleys, which guide a Dyneema rope fixed to the end of the shoulder elevation lever. Pre-tensioned springs are connected to the shoulder module and the Dyneema rope via a Bowden cable. If the shoulder joint angle changes, the spring tension and consequently the compensation torque changes. Gravity torque is not fully compensated so that the arm slowly moves down by gravity when relaxed.

The gravity compensation is designed for users with anthropometric measures between 5th female and 95th male percentiles. Fine tuning of the gravity support is done by a stepper motor combined with a timing belt and a driven thread that changes the pre-load of the spring package.

The weight relief for the forearm is also realized by a spring linked with a cable pull, which is manually adjusted. The applied compensation torque at the elbow does 


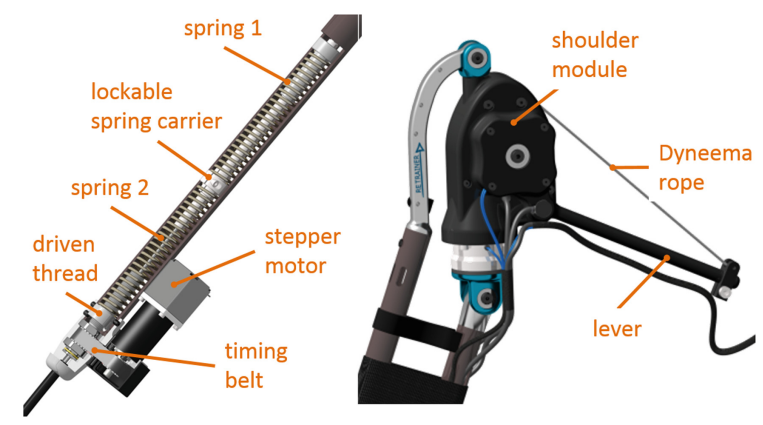

Fig. 2. Shoulder module with external compensation spring unit. For higher gravity compensation, it is possible to change from two-spring to one-spring mode. Changing to one-spring mode results in a higher total spring stiffness and consequently in a higher gravity compensation torque at the shoulder joint.

not change with upper arm position. For movement sequences with elevated upper arm and flexion/extension of the elbow in a more or less horizontal plane where gravity does not have a large impact on the movement, the compensation can be disabled by inserting a clip.

\subsection{Experimental Setup and Measurements}

Measurements were done to determine the effectiveness of the gravity support. Trajectories of 8 reflective markers, placed on the subject's torso and left upper limb, were collected with a camera motion analysis system (Motion Analysis Corporation). Electromyographic (EMG) signals were recorded with a wireless EMG signal detection system (Delsys Trigno Lab), the electrodes were placed on the skin above the muscles Deltoideus ant., Deltoideus med., Deltoideus post. and Biceps brachii. Maximum isometric joint torques were recorded using a force sensor (K6D40, ME Messsysteme).

Five healthy subjects were instructed to carry out two different motions - shoulder elevation in parasagittal plane and shoulder elevation in frontal plane (elbow neutral) with an additional weight of $0.5 \mathrm{~kg}$ in the hand to enhance muscle excitations. Each movement was repeated three times. Two sets of data were recorded, one with enabled and one with disabled gravity compensation. The gravity compensation was adjusted so that gravity slowly moved the fully extended arm (neutral elbow joint angle) downwards from an initial shoulder elevation angle of $90^{\circ}$. For each subject maximum isometric joint torques and maximum voluntary contractions (MVC) of shoulder muscles and biceps were measured in isometric test routines [4].

\subsection{Simulation}

For performing the simulations and estimating muscle activations and forces the software package OpenSim was used [5]. From motion data joint angle trajectories for each movement were computed using the Inverse Kinematics tool. The computed muscle control (CMC) algorithm [6] was then used to determine muscle forces and activations. 


\section{Results and Discussions}

Experiments on the RETRAINER arm exoskeleton have shown, that muscle activations could be significantly reduced during shoulder elevation movements when gravity compensation was enabled. Muscle activations determined by the simulations showed similar behavior as the measured EMGs, indicating the validity of the musculoskeletal model. The estimated muscle forces (Fig. 3) indicate highest rates of force reduction due to the gravity compensation between $20^{\circ}-80^{\circ}$ of shoulder elevation $(60 \% \mathrm{Del}$ toideus ant., 80\% Deltoideus med., Deltoideus post. 75\%).

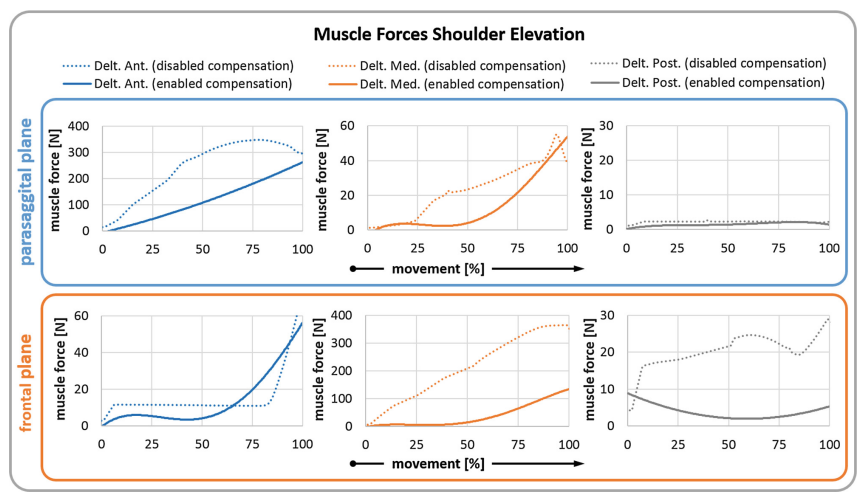

Fig. 3. Comparison of predicted shoulder muscle forces from simulation for enabled (full lines) and disabled (dashed lines) gravity compensation for one subject as a representation of the whole group.

The region between $20^{\circ}-80^{\circ}$ of shoulder elevation is optimal for rehabilitation and most activities of daily life. For an application as gravity compensating device for overhead work, modifications of the shoulder joint module would be necessary to move the region of optimal compensation to higher elevation angles. A body shell for mobile use has been developed and tested, which allows to mount the exoskeleton directly on the trunk and fix it at the hip similar to a backpack. However, as [7] have shown, also loads on body parts that are not the primary design focus of the technology have to be considered for safe application.

\section{Conclusion and Outlook}

Beside its compact and lightweight design, this study indicates that the gravity compensation of the RETRAINER exoskeleton can significantly reduce required muscle forces for arm movements. This exo was primarily developed for effective support in the rehabilitation process, but due to its modular structure, it can, with minor adaptions, also effectively be used to avoid work-related musculoskeletal disorders, especially in case of overhead work. 


\section{References}

1. Bengtson, S.: Early skeletal fossils. Paleontol. Soc. Pap. 67-77 (2004)

2. Rashedi, E., et al.: Ergonomic evaluation of a wearable assistive device for overhead work. Ergonomics 57, 1864-1874 (2014)

3. Puchinger, M., et al.: The ReTrainer arm exoskeleton - a modular lightweight device that combines adjustable gravity compensation, lockable joints \& NMES. In: Proceedings of the 12th Vienna International Workshop on FES, pp. 11-14 (2006)

4. Douma, R.K.W., et al.: Reference values for isometric muscle force among workers for the Netherlands: a comparison of reference values. BMC Sports Sci. Med. Rehabil. 6(1) (2014)

5. Delp, S.L., et al.: OpenSim: open-source software to create and analyze dynamic simulations of movement. IEEE Trans. Biomed. Eng. 54(11), 1940-1950 (2007)

6. Holzbaur, K.R.S., et al.: A model of the upper extremity for simulating musculoskeletal surgery and analyzing neuromuscular control. Ann. Biomed. Eng. 33(6), 829-840 (2005)

7. Kim, S., et al.: Assessing the influence of a passive, upper extremity exoskeletal vest for tasks requiring arm elevation: Part II - "Unexpected" effects on shoulder motion, balance, and spine loading. Appl. Ergon. (2018). epub ahead of print 